\title{
Effect of Ce on Cast and Extruded Microstructure of ZK20 Alloy Quan $\mathrm{Li}^{1, \mathrm{a}}$, Weibo Zhu ${ }^{1, \mathrm{~b}}$, Bin Zeng ${ }^{1,2, \mathrm{c}}$, Xianquan Jiang ${ }^{1, \mathrm{~d}}$ \\ ${ }^{1}$ Chongqing Academy of Science and Technology, Chongqing 401123, China \\ ${ }^{2}$ College of Materials Science and Engineering, Chongqing University, Chongqing 400044, China

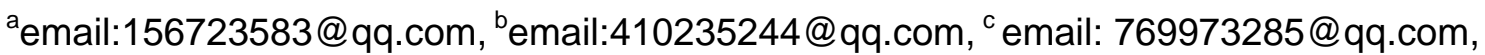 demail:574754298@qq.com
}

Keywords: Magnesium Alloy, Cast, Extrusion, Microstructure;

\begin{abstract}
In this paper, Effect of different contents of Ce on ZK20 magnesium alloy by conventional metallographic, X-ray, SEM, XRD, analysis of Ce element exists in the form of ZK20 magnesium alloy and microstructure of action the results show that: in the experimental range ( $0 \%$ $\sim 0.68 \% \mathrm{Ce}$ (mass fraction)), with the addition of Ce, appearing in the cerium-containing alloy phase $\tau$ growing phase, not to form a continuous network and net change, after the alloy by hot extrusion, the alloy of cerium can improve the degree of recrystallization, the grain size increases with the amount of cerium increasingly smaller.
\end{abstract}

\section{Introduction}

Increasing human demand for energy, and the next few decades the world's oil output may reach the limit, prompting people to consider lowering the vehicle in response to depend petroleum-based fuels. Car weight is an important initiative, weight $40 \mathrm{~kg}$ and recognized experience based on vehicle mass reduced by $10 \%$, savings of about $7 \%$. Magnesium is the lightest structural metal, an alloy is an ideal material for automotive weight reduction, in North America, Europe and China, the magnesium in automotive applications is increasing. However, application of magnesium alloy in the car is also largely remain in the monomer component level, and limited quality castings mainly before magnesium alloy in the form of local assembly used in automobiles, automobile magnesium as the main structural material in reduce vehicle mass and improve performance of the role of cars is not yet fully reflected. This requires further development of new materials and deformation of magnesium alloy magnesium extrusion, sheet metal forming, casting high integration, connecting and assembly-based manufacturing process technology, including low-cost high-performance new materials development wrought magnesium alloy, has become a concern one focus ${ }^{[1-5]}$.

\section{Experimental}

Water-cooled semi-continuous casting system with different concentrations of Ce casting ZK20 magnesium alloy semi-continuous slab containing the chemical composition of each alloy in Table 1.The resulting sample was cast in the wind circulation of $12 \mathrm{KW}$ box-type resistance furnace $420^{\circ} \mathrm{C}$ $\times 10 \mathrm{~h}$ homogenizing annealing treatment, ingot peeling, and extrusion tests in 500 tons horizontal extruder, extrusion, in the blank mold holding furnace to $400^{\circ} \mathrm{C} \times 2 \sim 3 \mathrm{~h}$, the extruder barrel set temperature was $390^{\circ} \mathrm{C}$, the extrusion speed is $3 \sim 5 \mathrm{~m} / \mathrm{min}$. The microstructure of the sample on the OLYMPUS metallurgical microscope, metallurgical samples with $4.0 \%$ alcohol solution of nitric acid corrosion, with a scanning electron microscope XL30-TMP-type fracture morphology. Using RigakuD/max-1200 X-ray diffractometer ( $\mathrm{Cu}$ target, $\mathrm{K} \alpha$ ) performed phase analysis. TESCAN company VEGA II LMU using variable vacuum SEM and EDS were scanned and morphology compound component analysis. 
Table 1 Chemical compositions of experimental alloys (mass fraction, \%)

\begin{tabular}{|c|c|c|c|c|c|c|}
\hline Alloy code & $\mathrm{Si}$ & $\mathrm{Fe}$ & $\mathrm{Zn}$ & $\mathrm{Zr}$ & $\mathrm{Ce}$ & $\mathrm{Mg}$ \\
\hline ZK20 & 0.0063 & 0.0046 & 2.11 & 0.021 & 0.00 & $\mathrm{Bal}$ \\
\hline ZK20+0.1Ce & 0.0062 & 0.0038 & 2.05 & 0.023 & 0.09 & Bal \\
\hline ZK20+0.3Ce & 0.0061 & 0.0020 & 2.07 & 0.021 & 0.28 & Bal \\
\hline ZK20+0.5Ce & 0.0065 & 0.0018 & 2.06 & 0.062 & 0.47 & Bal \\
\hline ZK20+0.7Ce & 0.0058 & 0.0016 & 1.98 & 0.045 & 0.68 & Bal \\
\hline
\end{tabular}

\section{Results and Analysis}

Alloy as-cast organization of SEM morphology as shown in figure 1 (a) (e), as shown in the figure shows that as-cast ZK20 alloy dendrites is very developed, granular exists between the dendrite alloy phase, with the increase of cerium content, the number of alloy phase interdendritic gradually increased, the continuity of alloy phase, dendrites become more clear, when cerium adding amount was $0.3 \%$, alloy phase morphology to mesh and discontinuous reticular shift, ZK20+0.5Ce alloy in the alloy phase has formed obvious mesh.

Alloy homogenization SEM rupture as shown in figure 1 (f) to (j), as shown in the figure shows that ZK20 after homogenizing annealing, grain boundary clear, originally exists in interdendritic granular completely solid solution alloy phase in the matrix. With the increase of cerium content, part of the alloy phase solid solution to the matrix, but there are still some alloy phase at grain boundary residual, the grain size of alloy decreases, and the residue of the alloy to meet a certain effect on dynamic recrystallization.
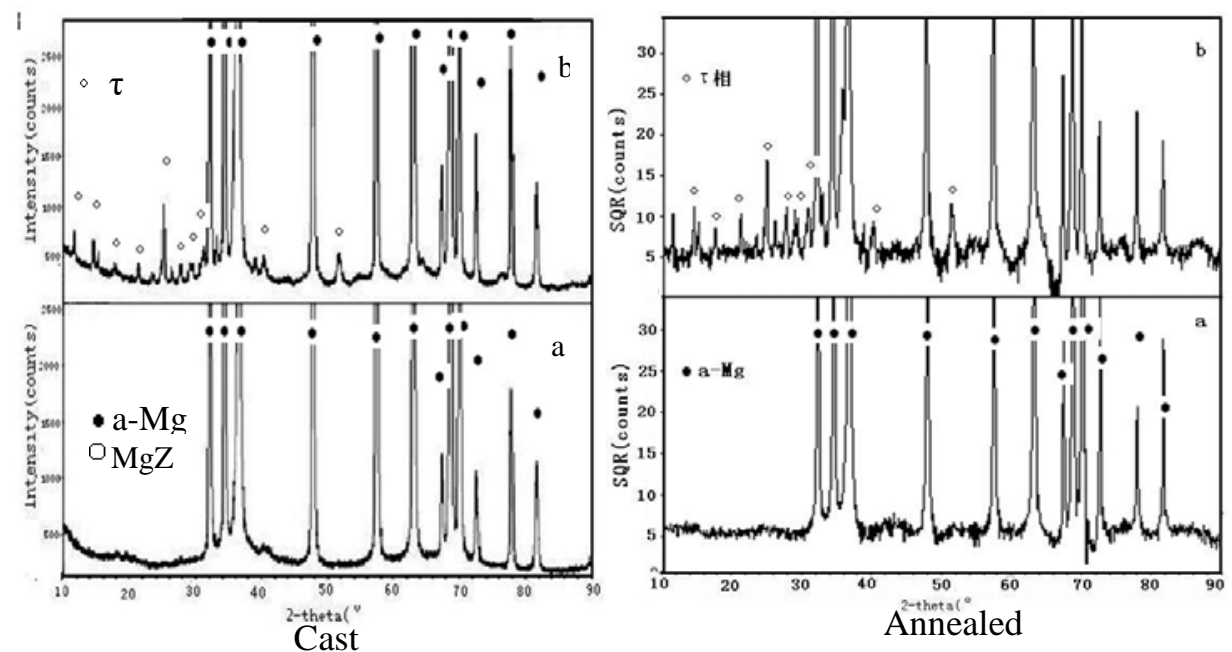

Figure2. X-Ray diffraction Patterns of as-cast amd as-annealed alloys (a)ZK20; (b)ZK20+0.7Ce

Alloy extrusion organization as shown in figure $1(\mathrm{k}) \sim(\mathrm{o})$, as shown in the figure shows that squeezed state ZK20 alloy uneven grain size, the local has a chain of fine grain structure, that is a reflection of recrystallization is not sufficient. Under the same conditions, cerium in more than $0.1 \%$ of the alloy, the grain size is evener, recrystallization adequately. With the increase of cerium content, grain size of alloy has the tendency of further decrescent, by $29.6 \mathrm{mu}$ to $15.3 \mathrm{mu}$, alloy in the process of solidification, cerium composition undercooling role, in the process of extrusion, the solution to the substrate of cerium on the grain refinement. 


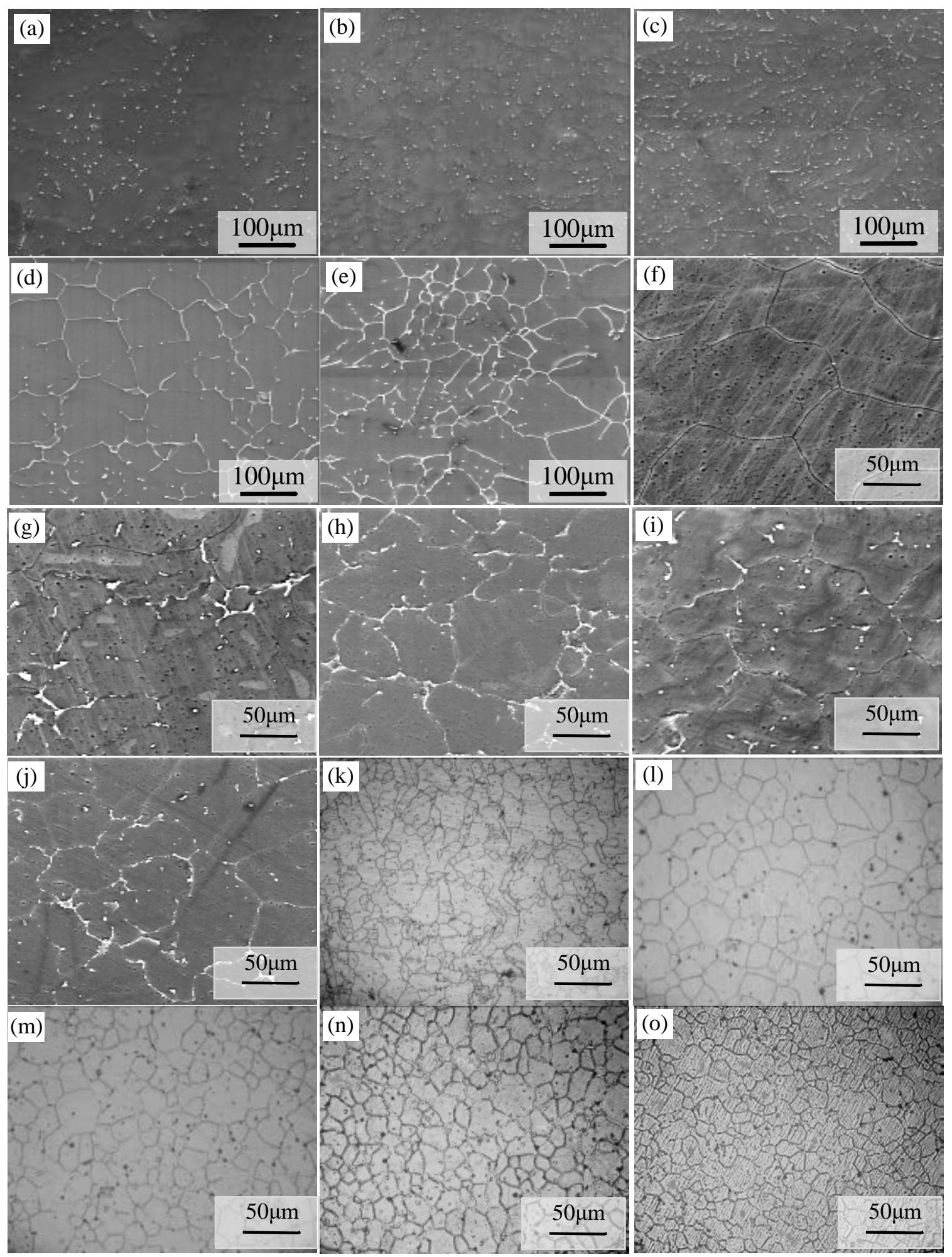

Figure1.The as-cast and as-annealed SEM microstructure of alloys and extruded alloy microstructure (a): SEM image, ZK20 alloy, as-cast; (b): SEM image, ZK20+0.1Ce alloy, as-cast;(c): SEM image, ZK20+0.3Ce alloy, as-cast; (d): SEM image, ZK20+0.5Ce alloy, as-cast;(e): SEM image, ZK20+0.7Ce alloy, as-cast; (f): SEM image, ZK20 alloy, as-annealed; (g): SEM image, ZK20+0.1Ce alloy, as-annealed; (h): SEM image, ZK20+0.3Ce alloy, as-annealed; (i): SEM image, ZK20+0.5Ce alloy, as-annealed; (j): SEM image, ZK20+0.7Ce alloy, as-annealed; .(k): Optical microstructure, ZK20 alloy, as-extruded; (l): Optical microstructure, ZK20+0.1Ce alloy, as-extruded;(m): Optical microstructure, ZK20+0.3Ce alloy, as-extruded; (n): Optical microstructure, ZK20+0.5Ce alloy, as-extruded; (o): Optical microstructure, ZK20+0.7Ce alloy, as-extruded;

As-cast and homogenizing annealing state alloy analysis XRD spectra as shown in figure 2, as shown in the figure shows that without adding Ce alloy elements ZK20 alloy as-cast organization for a-Mg matrix and granular Mg-Zn phase, adding Ce alloy elements ZK20+0.7Ce alloy phase 
mainly tau phase from the alloy. Will ZK20 and ZK20+0.7Ce alloy after homogenizing annealing condition, the alloy phase changed, including ZK20 alloy after homogenizing annealing, Mg-Zn phase in the organization of the diffraction peak disappeared; ZK20+0.7Ce tau in the alloy phase still exists, the diffraction peak tau phase has not completely solid solution in the matrix, the kinds of alloy phase does not change with the homogenizing annealing.

\section{Conclusion}

ZK20 magnesium alloy organization is composed of a-Mg, Mg-Zn phase, Mg-Zn phase existed between dendrite. With the increase of cerium content, emerge in the alloy containing cerium alloy phase tau phase, it is growing along with the increase of cerium content, form and to discontinuous mesh and mesh.

Within the scope of the study, cerium adding cannot be increased the strength of the as-cast alloy and plastic. With the increase of cerium content, under the condition of non equilibrium solidification tau produce too much of it as reticulation or discontinuous reticular distribution, make the grain boundary become the main carrier of cracks produced and extended, grain boundary weakening. Can't coordinate deformation in the process of deformation, the grain boundary, and give priority to cracking, which makes the alloy with the increase of cerium, the strength of the alloy decreases and the plastic.

Alloy after hot extrusion, the dynamic recrystallization happens, do not contain cerium alloy incomplete recrystallization, grain size difference is bigger. Adding cerium alloy, recrystallization grain fine uniform, and with the increase of cerium content grain size is smaller. Tau squeezed state alloy phase is small granular.

\section{Acknowledgements}

This work is supported by Chongqing “ 121 ” Project (cstc2014zktjccxBX0078, cstc2014zktjccxBX0033).

\section{References}

[1] Yu Kun, Li Wenxian, Zhang Shijun. Mechanism of Grain Refining by Adding Cerium in Mg and Mg Alloys[J]. Rare Metal Materials and Engineering, 2005, Vol.34(7):1013-1016.

[2] Li Wenxian. Magnesium and magnesium alloys[M]. Changsha:Central South University Press, 2005.6.

[3] Pan Fusheng, Han Enhou. High-Performance Wrought Magnesium Alloy and Their Processing Technigues[M]. Beijing: Science Press, 2007.18.

[4] GEIGER M, MERKLEIN M. Sheet metal forming-a new kind of forge for the future [J].Key Eng Mater, 2007, 344:39-46.

[5] ZENG Xiaoqin, DING Wenjiang, YAO Zhengyu, et al. The microstructure and mechanical properties of Mg-Zn-Al alloys[J]. Journal of Shanghai Jiaotong University, 2005, 39 (1): 46-51. 